Revue des patrimoines

$31 \mid 2017$

Patrimoines de la santé : essais de définition - enjeux de conservation

\title{
Une réflexion sur la forme des salles d'hôpital : le mémoire du médecin Hugues Maret et son illustration par Jacques-Germain Soufflot
}

A reflection on the form of hospital wards: the memoir of the doctor Hugues

Maret and its illustrations by Jacques-Germain Soufflot

\section{Marc Lauro}

\section{CpenEdition}

\section{Journals}

Édition électronique

URL : http://journals.openedition.org/insitu/13914

DOI : 10.4000/insitu.13914

ISSN : 1630-7305

\section{Éditeur}

Ministère de la Culture

\section{Référence électronique}

Marc Lauro, «Une réflexion sur la forme des salles d'hôpital : le mémoire du médecin Hugues Maret et son illustration par Jacques-Germain Soufflot », In Situ [En ligne], 31 | 2017, mis en ligne le 21 février 2017, consulté le 09 octobre 2020. URL : http://journals.openedition.org/insitu/13914 ; DOI : https:// doi.org/10.4000/insitu.13914

Ce document a été généré automatiquement le 9 octobre 2020.

\section{c) (i)}

In Situ Revues des patrimoines est mis à disposition selon les termes de la licence Creative Commons Attribution - Pas d'Utilisation Commerciale - Pas de Modification 4.0 International. 


\title{
Une réflexion sur la forme des salles d'hôpital : le mémoire du médecin Hugues Maret et son illustration par Jacques-Germain Soufflot
}

\author{
A reflection on the form of hospital wards: the memoir of the doctor Hugues \\ Maret and its illustrations by Jacques-Germain Soufflot
}

Marc Lauro

\section{Introduction}

1 À la fin du xvIII ${ }^{\mathrm{e}}$ siècle fleurit une littérature médicale ${ }^{1}$ qui envisage l'hôpital non plus comme une institution charitable qui prend en charge les pauvres et les malades mais comme un établissement de soins conçu par des médecins ou des physiciens ${ }^{2}$. Le texte d'Hugues Maret ${ }^{3}$ illustre parfaitement cette évolution: un médecin et académicien décrit la meilleure forme à donner à une salle d'hôpital selon les critères hygiénistes de l'époque et un architecte, en l'occurrence Jacques-Germain Soufflot, fournit le plan qui illustre ce traité.

\section{Le contexte : «l'affaire des hôpitaux »}

2 Le mémoire du médecin dijonnais Hugues Maret sur la meilleure forme à donner à une salle d'hôpital s'inscrit dans un débat qui, de 1772 à 1789, devait mobiliser les sociétés savantes de province et l'Académie royale des sciences. Après un premier incendie dévastateur en 1737, l'hôtel-Dieu de Paris brûle à nouveau en 1772 et le gouvernement royal décide alors de nommer deux commissions, l'une présidée par Necker en 1772 et l'autre par le baron de Breteuil en 1785. L'Académie royale d'architecture n'est pas consultée à cette occasion. Largement composées par des médecins et des physiciens 
tels Tenon ou Lavoisier, ces commissions visitent les établissements hospitaliers pour en repérer les défauts et réceptionnent les projets qui leur sont soumis. Les plans de reconstruction de l'Hôtel-Dieu ne s'appuient plus sur des références architecturales antérieures mais sur les idéaux médicaux et disciplinaires du Siècle des Lumières. Le travail d'enquête est mené, en grande partie, par le chirurgien Jacques Tenon qui publie le résultat de son travail en $1788^{4}$. Il se rend dans plusieurs hôpitaux français et anglais avec le physicien Charles-Augustin Coulomb ${ }^{5}$, collecte des plans d'architectes et examine les nombreux mémoires qui sont publiés sur ce sujet. Ces mémoires sont l'aboutissement de l'association d'un médecin ou d'un physicien, pour les principes généraux, avec un architecte, pour le plan. Conservés aux archives de l'Assistance publique, ils donnent une image précise de la pensée hygiéniste des années 1780 , en même temps qu'ils témoignent d'une des premières enquêtes publiques destinées à l'édification d'un équipement collectif. Le projet du médecin Maret ne fut pas retenu par la commission des hôpitaux mais son illustration par Soufflot et son caractère novateur en font un des ouvrages les plus originaux sur ce sujet.

3 Il convient, pour mieux comprendre le texte de Maret, de distinguer trois termes confondus aujourd'hui dans leur acception contemporaine ${ }^{6}$. Au XvII ${ }^{e}$ siècle, un hôtelDieu accueille tous les malades, sans distinction, et sa gestion est assurée par l'Église. Un hôpital est quant à lui destiné à accueillir, ou plutôt enfermer, les pauvres, et son administration relève des municipalités ou du Parlement pour la ville de Paris. À la fin du XVIII ${ }^{\mathrm{e}}$ siècle, les auteurs commencent à utiliser le terme d'« hôpital » pour désigner indifféremment un établissement de soins; l'infirmerie est le dortoir des malades proprement dit. Ce glissement sémantique s'explique par le fait que les hôpitaux sont de plus en plus amenés à construire des infirmeries en leur sein. De façon implicite, le terme hôpital renvoie à une prise en charge des malades qui ne serait plus le monopole de l'Église, la bienfaisance publique se substituant à la charité chrétienne.

\section{Un médecin des Lumières}

4 C'est à la demande des administrateurs de l'hôpital d'Aix-en-Provence, sollicitant l'avis de leurs homologues dijonnais sur la meilleure façon d'assurer l'aération des infirmeries, que Maret fut amené en 1775 à concevoir une forme radicalement nouvelle de salle d'hôpital' ${ }^{7}$ Son itinéraire professionnel permet d'expliquer pourquoi il fait figure de spécialiste de la question.

5 Né en 1726 dans une famille de chirurgiens, il accède au rang de médecin et de professeur, ouvrant dès 1773 dans sa ville natale de Dijon un cours gratuit de chimie et de botanique.

6 Secrétaire perpétuel de l'académie de Dijon et correspondant de l'Académie des sciences de Paris, il publie de nombreux mémoires sur les bienfaits de l'inoculation de la vaccine ${ }^{8}$ et des bains de mer ${ }^{9}$ ainsi que sur la nécessité de transférer les cimetières hors des villes. Outre son activité d'auteur et de professeur, il fut un praticien très actif et succomba à la tâche en 1786, en tant que médecin des États de Bourgogne, alors qu'il tentait d'arrêter la propagation d'une «fièvre pestilentielle » qui décimait le village de Fresne-Saint-Mamès (Haute-Saône) ${ }^{10}$.

7 L'œuvre de Maret et sa collaboration avec Soufflot permettent de comprendre à la fois ce qu'était la pensée aériste, qui ne fut totalement remise en cause qu'avec les 
découvertes de Pasteur, en même temps qu'elles éclairent une prise de pouvoir théorique des médecins sur les architectes afin de concevoir, selon les mots du chirurgien Tenon, «une machine à guérir ${ }^{11} »$. Ce travail résulte également de la collaboration de deux Bourguignons qui ont œuvré dans leur province d'origine; c'est sans doute lors de l'édification, sur les plans de Soufflot, des établissements hospitaliers de Mâcon (Saône-et-Loire) ${ }^{12}$ que les deux hommes se sont rencontrés ou ont été amenés à correspondre. Secrétaire perpétuel de l'académie de Dijon et hygiéniste reconnu, Maret possède toute la légitimité nécessaire pour solliciter le concours de Soufflot dans son travail scientifique.

Publié le 10 avril 1780 dans Le Journal de Paris, l'essentiel de son mémoire est l'aboutissement, nous informe Maret, de sa correspondance avec Soufflot, qui avait travaillé au remaniement ou à la construction de trois hôpitaux : l'hôtel-Dieu de Lyon ${ }^{13}$, l'hospice de la Charité et l'hôtel-Dieu de Mâcon ${ }^{14}$. L'architecte, tenant compte du bâti existant, y reprenait alors les modèles traditionnels de l'architecture hospitalière: bâtiments en croix ou en carré délimitant des cours fermées et dont l'origine est à retrouver dans les constructions conventuelles puisque c'est encore très largement le clergé qui est à l'origine des fondations charitables. À ce titre, l'ospedale Maggiore de Milan ou l'hôpital Saint-Louis de Paris font l'objet de reproductions sous forme de plans détaillés dans les mémoires consacrés à l'architecture des hôpitaux, tel celui de Tenon. Notons que dans ses constructions, Soufflot s'applique à mettre en œuvre certaines des préoccupations aéristes des années 1770. Ainsi, le nouvel hôtel-Dieu de Macon est-il bâti sur un sol exhaussé, les salles de malades ont une hauteur de six à sept mètres, et sont éclairées par au moins six fenêtres élevées de 2,80 mètres permettant l'aération. La distinction entre salles de soins, services administratifs et salles de service y est nette, et la coupole de la chapelle, qui est à la jonction de trois corps de bâtiments, est censée permettre le renouvellement de l'air. Ce plan en croix, issu de l'architecture religieuse, est entièrement repensé par Maret dans son mémoire ${ }^{15}$. Soufflot transcrit dans le dessin gravé pour cet ouvrage la description du médecin. Publiée en 1782, soit deux ans après la mort de Soufflot, par l'académie des sciences de Dijon, sa planche ne figure que la coupe et le plan masse dessinés par l'architecte mais non pas le plan général des bâtiments, néanmoins décrit par Maret. Son caractère étonnamment épuré apparaît comme très éloigné des productions connues du concepteur de la basilique Sainte-Geneviève de Paris. Cela s'explique par le fait que Soufflot modèle ici l'espace en fonction des théories de Maret sur la circulation de l'air et renonce donc à tout effet décoratif. En outre, si Maret ne donne pas les détails de sa correspondance avec l'architecte, la gravure peut apparaître comme la mise au propre d'un croquis, une forme modelant l'invisible, cet air qui circule horizontalement à travers une salle de malades. On ne peut donc lire et comprendre cette gravure qu'à la lecture du texte qu'elle illustre, «d'après les vues de l'auteur » nous indique sa légende.

9 Le mémoire de Maret constitue en fait une démonstration logique en trois parties: partant de la nature de l'air, il en déduit son mode de circulation, expérimente sa théorie et l'applique à la construction d'un hôpital.

\section{Ce qui arrive à l'air renfermé dans les infirmeries}

10 Selon Maret, l'air est un fluide élastique auquel se mêlent toutes les substances volatiles qui l'environnent; ainsi, l'air situé à proximité d'un foyer d'infection se charge en 
particules «malsaines ». Dans une salle de malades, il se crée donc deux couches d'air de nature distincte. Au niveau des malades, on trouve une couche où stagnent les exhalaisons malsaines tandis qu'au niveau du plafond demeure un air non corrompu. L'air supérieur pesant sur l'air inférieur, ce dernier ne peut s'évacuer par les dômes ou les ventouses que l'on dispose au sommet des salles. Pour preuve, Maret décrit une expérience qu'il a menée à l'hôpital de Lyon. Il a disposé dans un des dômes de l'hôpital une cage d'oiseaux et a constaté, au bout de quinze jours, que les oiseaux sont toujours en bonne santé malgré le caractère prétendument mortel de l'air sous le dôme d'un hôpital. Il renouvelle l'expérience avec de la viande fraîche : à hauteur du lit des malades, la viande se corrompt en moins de vingt-quatre heures, à hauteur du dôme, elle n'est toujours pas corrompue au bout de cinq jours. Maret en déduit donc qu'il faut évacuer l'air au niveau des malades et non plus du plafond.

\section{Comment circule l'air dans une salle}

Pour expliquer le schéma de circulation de l'air, le médecin utilise l'exemple d'une rivière qui passe sous un pont et dont le courant devient visible. Celui-ci adopte la forme d'un cône au débouché des piles du pont et devient une ellipse en rencontrant à nouveau une résistance dans la masse d'eau plus calme qui borde les berges de la rivière. L'air qui pénètre dans une salle munie de fenêtres à chacune de ses extrémités adopterait donc la même forme.

Lorsque l'on ouvre les fenêtres, le renouvellement de l'air se fait horizontalement et selon un trajet elliptique. Or, aucune salle n'est alors conçue en fonction de ce déplacement de l'air : les lits situés le long des murs restent en dehors de ce courant et les malades continuent à stagner dans un air vicié.

\section{Principes de construction d'un hôpital}

13 Afin de permettre un renouvellement complet de l'air, Maret propose sa forme idéale que reprend le plan de Soufflot:

Je voudrais que les infirmeries eussent la forme d'une ellipse plus ou moins allongée et tronquée à chacun de ses foyers, que leur voûte fût également elliptique, et que la partie supérieure des murs se réunit à cette voûte par une courbure du même genre, de sorte que la salle eût la forme d'un œuf coupé par un plan parallèle au grand axe de la principale ellipse.

14 Pour faciliter le passage de l'air, le médecin prévoit l'utilisation de meubles portatifs que l'on enlève hors de la salle au moment de l'ouverture des fenêtres. L'autel, lui aussi portatif, devra être retiré de la salle lors de son aération ou au moment des opérations car il ne convient pas, selon lui de créer un temple là où « l'on crie de douleur ». Il est, à ce titre, caractéristique de la pensée des Lumières qui commence à distinguer charité chrétienne et bienfaisance publique, dévouement des congrégations hospitalières et approche scientifique de la maladie par le corps médical ${ }^{16}$.

Dans la disposition générale des bâtiments, son projet rompt également avec les modèles formels antérieurs. Il prévoit des infirmeries parallèles les unes aux autres et toutes orientées du nord au midi. Sept ans avant les conclusions de la commission des hôpitaux chargée de reconstruire l'hôtel-Dieu de Paris, il propose donc un plan qui aura une valeur séculaire: une disposition pavillonnaire où les infirmeries forment des 
barres parallèles. À l'aube de la pensée clinique, le malade n'y trouve pas un remède efficace mais le médecin y voit assurément un musée vivant des pathologies, une proposition de séparation et de classement des maladies.

L'autre originalité du plan (fig. 1), illustrée, celle-ci, par Soufflot, est l'abandon de la ventilation verticale: elle permet de récupérer le rez-de-chaussée comme salle de malades tout en prenant soin de l'élever de deux ou trois pieds au-dessus du sol. La ventilation est assurée par des fenêtres en forme d'entonnoir que Soufflot dessine, en vis-à-vis, aux deux extrémités de la pièce. Les petites fenêtres en forme de hublot, le long des murs, sont uniquement destinées à éclairer la salle. Cette analogie avec l'architecture navale ne doit pas étonner puisque la référence aux procédés de ventilation des navires est souvent utilisée par les mémoires sur les hôpitaux, en particulier par le physicien Duhamel du Monceau qui avait étudié ce sujet et préconisait l'établissement de ventouses dans les infirmeries ${ }^{17}$.

Figure 1

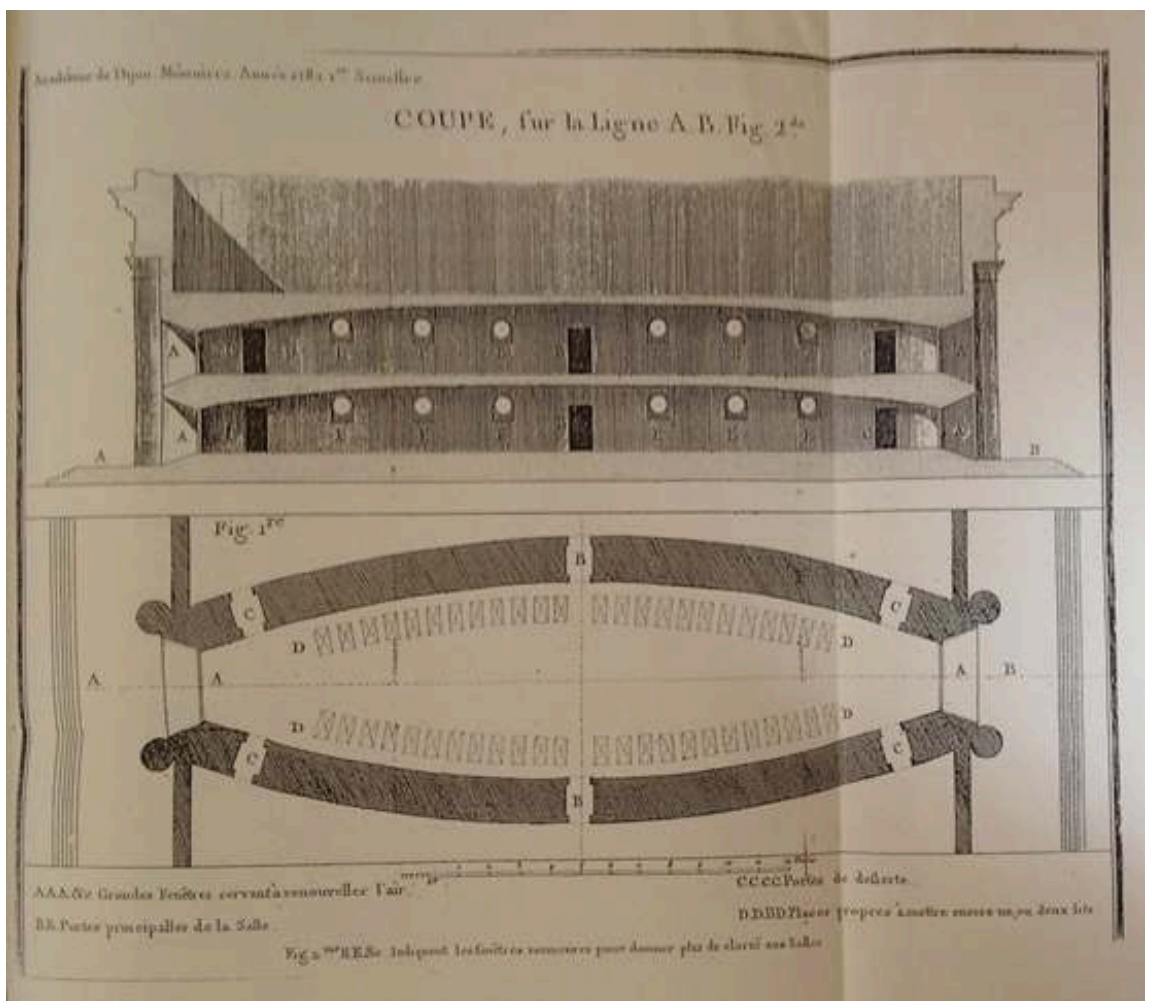

Planche extraite de l'ouvrage d'Hugues Maret. Mémoire sur la construction d'un hôpital dans lequel on détermine quel est le meilleur moyen à employer pour entretenir dans les infirmeries un air pur et salubre. Dijon : 1782.

Sur le plan, on peut observer des portes de desserte qui indiquent l'existence de pièces de service parallèles à l'infirmerie et permettent de séparer les flux de circulation autant que les flux d'air. C'est la même logique qui explique que le dernier étage soit entièrement dévolu au service, c'est-à-dire au stockage et à l'entretien des meubles et des draps. Comme emplacement pour son hôpital, Maret reprend également les préconisations des physiciens aéristes en choisissant un lieu " en plein air ", au nord et en dehors des villes, au bord d'une rivière, là où les flux circulent et s'évacuent librement. 


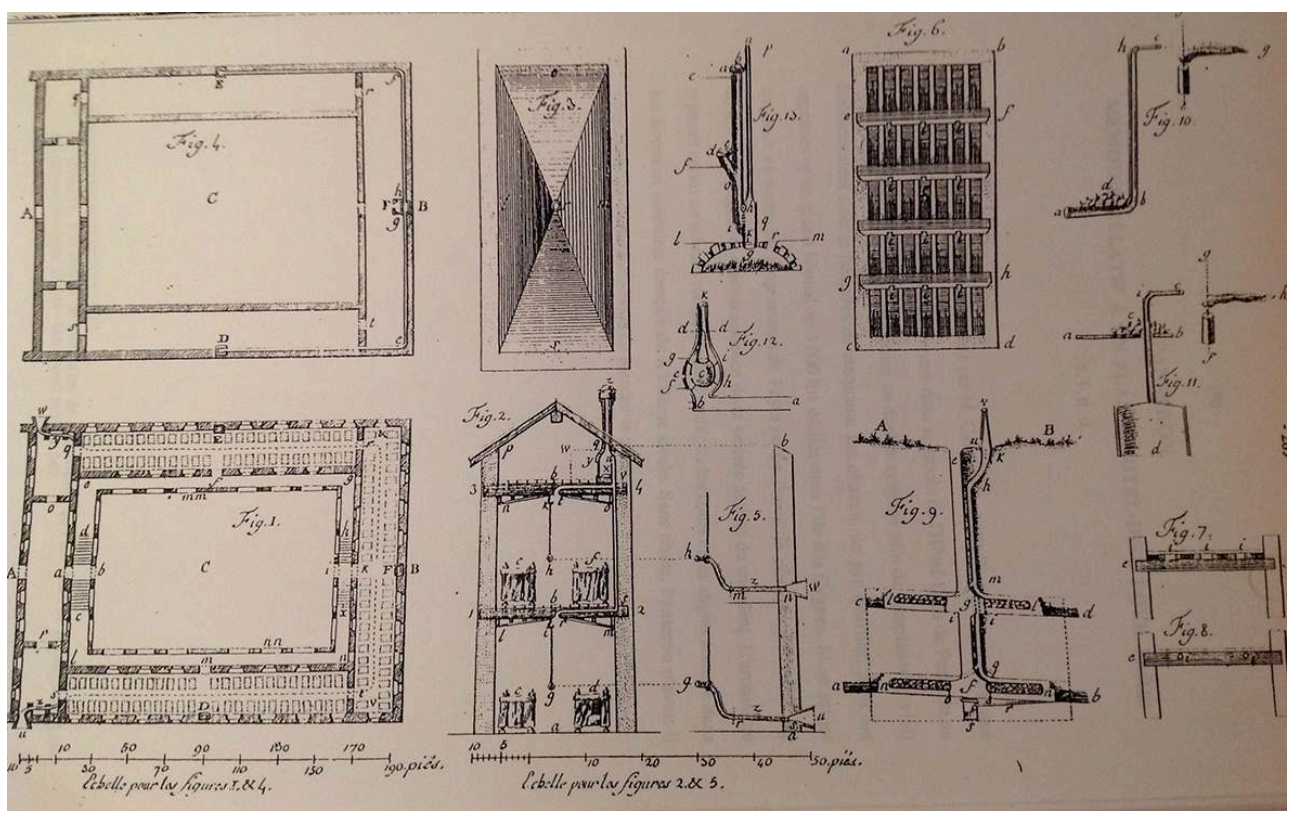

Planche extraite de l'ouvrage de Léopold de Genneté. Purification de l'air croupissant dans les hôpitaux, les prisons et les vaisseaux. Nancy : J.-B. H. Leclerc, 1767.

Maret conclut sa démonstration en rejetant deux projets contemporains. Il atteste ainsi de la circulation des idées dans les journaux savants et les académies de la fin du XVIII siècle et du dialogue qui s'établissait entre les savants par le biais des publications et des lectures lors des séances publiques des académies. Le premier projet (fig. 2), du physicien nancéen Léopold de Genneté, propose de purifier l'air en utilisant des poêles dans les infirmeries pour évacuer l'air vicié par des cheminées ${ }^{18}$. Le second projet, celui $\mathrm{du}$ professeur de médecine Antoine Petit (fig. 3), propose un hôpital de forme circulaire dont les salles disposées en rayons aboutiraient à une ventouse circulaire permettant de créer un flux continu d'air. Cette énorme machine pneumatique est conçue pour abriter de 1800 à 2400 malades, soit la capacité de l'hôtel-Dieu de Paris auquel il se substituerait ${ }^{19}$. Maret conteste dans ses deux projets la conception d'une circulation verticale de l'air avec une argumentation déjà évoquée mais il est intéressant de noter que ces mémoires font alors l'objet d'une double publication: d'abord dans les recueils d'articles des académies, parfois dans des journaux comme le Journal de Paris. À l'occasion, ils sont vendus séparément, sous forme de plaquettes tirésà-part afin de sensibiliser l'opinion publique à des sujets de société. Ici, le mémoire de Petit de 1774 et la réponse que lui apporte Maret alimentent le débat sur la reconstruction de l'Hôtel-Dieu. En effet, l'Académie des sciences doit d'abord informer les "gens éclairés » sur l'enquête en cours avant que le gouvernement ne finance ce projet. À cette fin, il doit lancer une large souscription en ces temps de déficit des finances de l'État, et alors que l'idée d'une assistance publique commence à se développer, remettant en cause la mission traditionnelle d'accueil des pauvres et des malades par l'Église. 
Figure 3

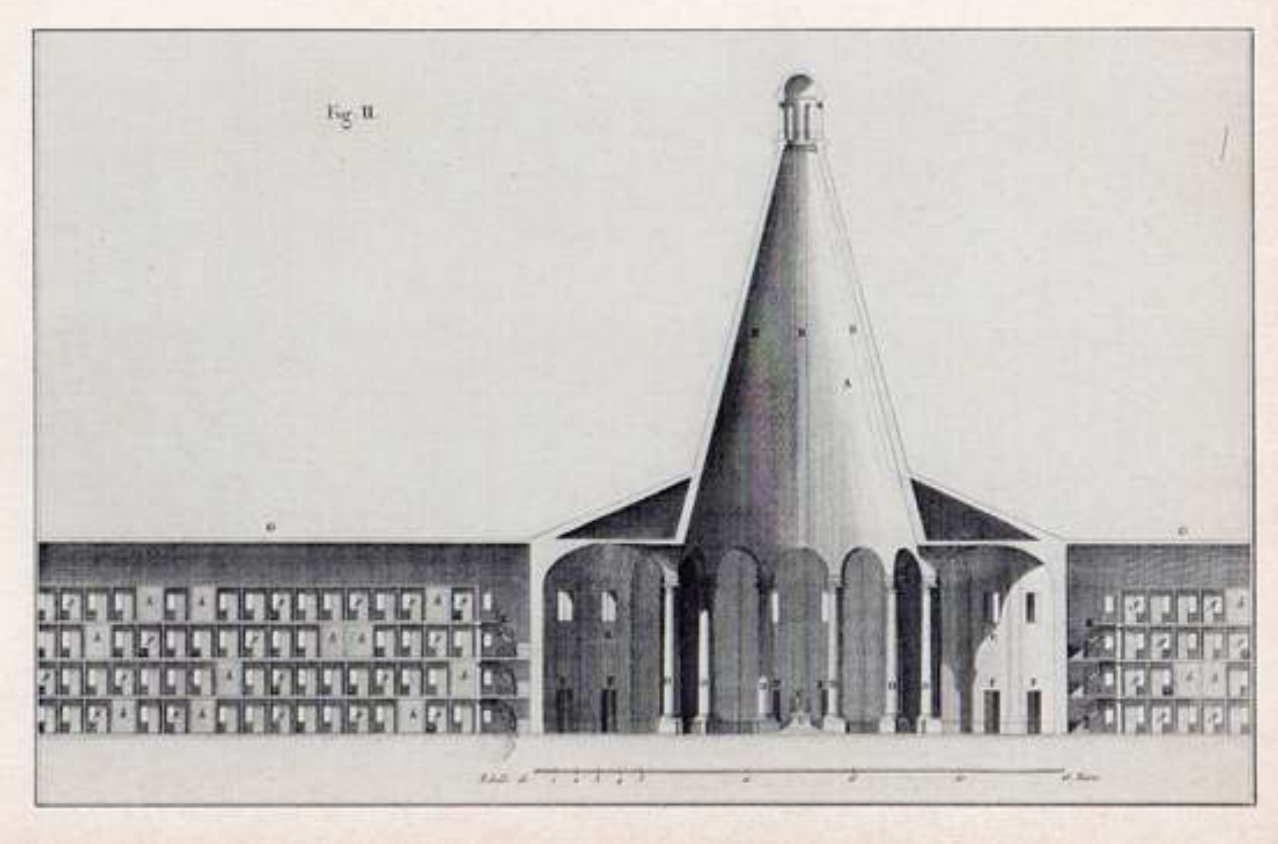

Planche extraite de l'ouvrage d'Antoine Petit. Projet et mémoire sur la meilleure manière de construire un hôpital de malades. Paris : Louis Cellot, 1774.

La mort de Soufflot ne permit pas de présenter un plan complet du programme de Maret mais la participation de cet architecte éminent est présentée par le médecin dijonnais comme la preuve évidente de la supériorité de son projet. Six ans avant les conclusions de l'Académie des sciences pour un projet de translation et de reconstruction de l'hôtel-Dieu de Paris (fig. 4), le mémoire de Maret anticipait sur deux acquis fondamentaux : la disposition en barres parallèles des infirmeries et l'abandon de l'aération verticale sous forme de dômes et de ventouses. Le temps des grandes machines panoptiques était révolu, au profit du calcul du cubage en oxygène nécessaire dans chaque salle de malades et du classement raisonné des pathologies dans des pavillons distincts. La Révolution interrompit le programme de construction de quatre hôpitaux à la périphérie de Paris en remplacement de l'Hôtel-Dieu mais la volonté de refonder l'hôpital comme un programme moderne et sur des considérations purement scientifiques perdura pendant un siècle. Les élites médicales de la monarchie de Juillet devaient se souvenir des leçons de Maret et de ses contemporains en posant la première pierre de l'hôpital Lariboisière ${ }^{20}$. 
Figure 4

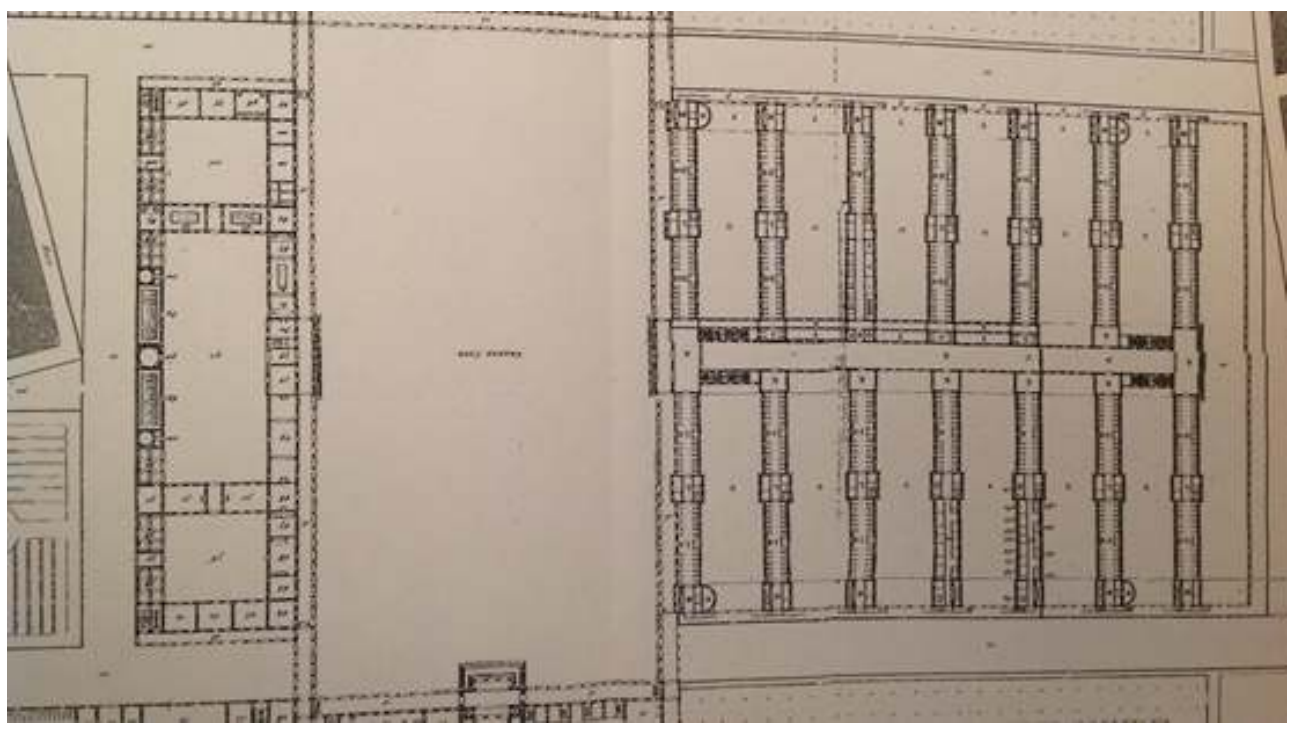

Plan d'un «hôpital de malades et de femmes enceintes » conçu par Jacques Tenon et dessiné par Bernard Poyet. Situé rue de la Roquette, cet établissement devait faire partie des quatre

établissements destinés à remplacer l'hôtel-Dieu de Paris. Jacques Tenon. Mémoires sur les hôpitaux de Paris. Paris : chez Royez, 1788, pl. XIV.

\section{NOTES}

1. - Une partie des plans accompagnant les mémoires consacrés à la reconstruction de l'HôtelDieu de Paris furent publiés par une équipe de chercheurs sous la direction de Michel Foucault à la fin des années 1970: THALAMY, Anne, KRIEGEL, Blandine, FOUCAULT, Michel, et al. Les machines à guérir. Aux origines de l'hôpital moderne. Bruxelles/Liège : P. Mardaga, 1979.

2. - «Émergence du programme pavillonnaire après l'incendie de l'hôtel-Dieu de Paris ». Dans : LAGET, Pierre-Louis, LAROCHE, Claude, DUHAU, Isabelle (dir.). L'hôpital en France: histoire et architecture. Lyon : éd. Lieux Dits (Cahiers du Patrimoine, n 99), 2012, p. 111.

3. - MARET, Hugues. " Mémoire sur la construction d'un hôpital dans lequel on détermine quel est le meilleur moyen à employer pour entretenir dans les infirmeries un air pur et salubre». Académie des sciences, arts et belles-lettres. Nouveaux mémoires de l'académie de Dijon pour la partie des sciences et des arts, 1782-1785, $1^{\mathrm{er}}$ semestre 1782, p. 25-67. Ce texte publié par l'Académie des Sciences sous la forme d'une plaquette en 1782, fut également publié par le Journal de Paris du 10 avril 1780. La réponse de Soufflot parut dans le même journal le 21 juin 1780 , soit deux mois avant sa mort.

4. - TENON, Jacques. Mémoires sur les hôpitaux de Paris. Paris, chez Royez, 1788.

5. - LAGET, Pierre-Louis. «Émergence du programme pavillonnaire après l'incendie de l'hôtelDieu de Paris ». Dans L'hôpital en France : histoire et architecture. Op. cit., p. 111.

6. - GUTTON, Jean-Pierre. "Réformes, projets et réalités à la fin de l'Ancien Régime». Dans IMBERT, Jean (dir.). Histoire des hôpitaux. Toulouse : Privat, 1982, p. 223-263.

7. - Ibid, p. 261-262. 
8. - MARET, Hugues. «Essais sur les fièvres épidémiques... lus les 29 janvier et 5 février 1762 ». Mémoires de l'Académie de Dijon, t. $1^{\mathrm{er}}, 1769$ (publiés à part en 1775).

9. - MARET, Hugues. « Mémoire sur la manière d'agir des bains d'eau douce et d'eau de mer et sur leur usage ». Mémoires de l'Académie de Dijon, 1767 (publié à part: Paris : Des Ventes de Ladoué, 1769).

10. - MICHAUD, Louis-Gabriel (dir.) Biographie universelle ancienne et moderne... Paris : ThoisnierDesplaces puis Michaud puis Brockhaus puis Desplaces, 1843-18..., t. 26,1865, p. 527.

11. - L'expression de Tenon fut reprise par François Béguin dans «La machine à guérir », l'article qu'il consacre à la naissance de l'hôpital comme équipement médical dans l'ouvrage du même nom : Les machines à guérir, aux origines de l'hôpital moderne, Op. cit., p. 39-43.

12. - PÉROUSE de MONTCLOS, Jean-Marie. Jacques-Germain Soufflot. Paris : éd. du Patrimoine, 2004. 13. - ROUBERT, Jacqueline. "L'hôtel-Dieu de Lyon au XVIII e siècle ». Dans TERNOIS, Daniel (éd.). Soufflot et l'architecture des Lumières. Paris : ministère de l'Environnement et du Cadre de vie, direction de l'Architecture/CNRS, 1980, p. 134-141.

14. - GARMIER, Jean-François. "Soufflot et Mâcon : la Charité, l'Hôtel-Dieu ». Dans Soufflot et l'architecture des Lumières. Op. cit., p. 142-151.

15. - MARET, Hugues. « Mémoire sur la construction d'un hôpital... ». Art. cit.

16. - LAGET, Pierre-Louis. «Aux origines de l'hôpital moderne, une évolution européenne ». Les Tribunes de la santé, 2004/2 (n³), p. 19-28.

17. - DUHAMEL du MONCEAU, Henri-Louis. Moyens de conserver la santé aux équipages des vaisseaux. Paris : chez H. L. Guérin et L. F. Delatour, 1759.

18. - GENNETÉ, Claude-Léopold. Purification de l'air croupissant dans les hôpitaux, les prisons et les vaisseaux. Nancy : J.-B. H. Leclerc, 1767.

19. - PETIT, Antoine. Mémoire sur la meilleure façon de construire un hôpital de malades. Paris : Louis Cellot, 1774.

20. - SAINTE FARE GARNOT, Nicolas, MARTEL, Pierre. L'architecture hospitalière au XIX ${ }^{e}$ siècle. Paris : ministère de la Culture/éd. de la Réunion des musées nationaux (Les dossiers du musée d'Orsay) : 1988, p. 32-43.

\section{RÉSUMÉS}

À la suite de l'incendie de l'hôtel-Dieu de Paris, en 1772, le gouvernement royal confie à l'Académie des sciences la mission de concevoir un nouvel établissement selon des normes médicales. Le mémoire du médecin Hugues Maret, illustré par Jacques-Germain Soufflot, s'inscrit dans cette réflexion autour de la meilleure forme à donner à un établissement de soins. Il permet de décrire ce qu'est alors la pensée aériste prépastorienne : l'air en mouvement participe de la guérison des malades et devient mortel quand il stagne. Ce texte annonce également les évolutions du XIX ${ }^{\mathrm{e}}$ siècle qui feront de l'hôpital un équipement collectif et une «machine à guérir » au service de la population.

Following the fire that destroyed the Paris Hôtel-Dieu in 1772, the government entrusted the Académie des Sciences with the task of designing a new establishment according to the medical norms of the day. The memoir of the doctor Hugues Maret, illustrated by Jacques-Germain Soufflot, is a part of this debate about the best form to be given to hospital buildings. It allows an analysis of what may be called pre-pasteurian aerism: air in movement contributes to the healing 
processes and becomes mortal if it stagnates. The text announces other evolutions during the nineteenth century which will make the hospital a collective facility and a 'healing machine' in the service of the population.

INDEX

Mots-clés : aérisme, commission des hôpitaux, hôtel-Dieu de Paris, académie des sciences de Dijon, Hugues Maret, Jacques-Germain Soufflot, Jacques Tenon

Keywords : aerisme, hospital commision, Hôtel-Dieu de Paris, Dijon academy of science, Hugues Maret, Jacques-Germain Soufflot, Jacques Tenon

\section{AUTEUR}

\section{MARC LAURO}

Doctorant à l'université Paris I, ED 441 lauromarc@yahoo.fr 